\title{
Non-adiabatic pulsations in ESTER models
}

\author{
Daniel Roy Reese ${ }^{1, \star}$, Marc-Antoine Dupret $^{2}$, and Michel Rieutord ${ }^{3,4}$ \\ ${ }^{1}$ LESIA, Observatoire de Paris, PSL Research University, CNRS, Sorbonne Universités, UPMC Univ. Paris 06, Univ. Paris \\ Diderot, Sorbonne Paris Cité, 92195 Meudon, France \\ ${ }^{2}$ Institut d'Astrophysique et Géophysique de l'Université de Liège, Allée du 6 août 17, 4000 Liège, Belgium \\ ${ }^{3}$ Université de Toulouse, UPS-OMP, IRAP, 31028 Toulouse, France \\ ${ }^{4}$ CNRS, IRAP, 14 avenue Edouard Belin, 31400 Toulouse, France
}

\begin{abstract}
One of the greatest challenges in interpreting the pulsations of rapidly rotating stars is mode identification, i.e. correctly matching theoretical modes to observed pulsation frequencies. Indeed, the latest observations as well as current theoretical results show the complexity of pulsation spectra in such stars, and the lack of easily recognisable patterns. In the present contribution, the latest results on non-adiabatic effects in such pulsations are described, and we show how these come into play when identifying modes. These calculations fully take into account the effects of rapid rotation, including centrifugal distortion, and are based on models from the ESTER project, currently the only rapidly rotating models in which the energy conservation equation is satisfied, a prerequisite for calculating non-adiabatic effects. Non-adiabatic effects determine which modes are excited and play a key role in the near-surface pulsation-induced temperature variations which intervene in multi-colour amplitude ratios and phase differences, as well as line profile variations.
\end{abstract}

\section{Introduction}

The interpretation of pulsation spectra in rapidly rotating stars has proven to be a considerable challenge. Indeed, due to the lack of simple and easily identifiable pattern in the pulsation spectra, it has been very difficult to establish the correspondence between observed pulsation frequencies and theoretically calculated modes, i.e. to carry out mode identification [1]. This is extremely problematic since it means we do not know what regions of the star are being probed by individual modes, and cannot, accordingly, carry out meaningful inversions of the stellar structure. Nonetheless, [2] and [3] have recently shown the presence of patterns in the period spacings of rapidly rotating $\gamma$ Dor stars, which agree with the most recent theoretical expectations [4-6] and allow us, in some cases, to determine the dominant harmonic degree and azimuthal order of these modes. For $\delta$ Scuti stars, progress remains more limited. In a recent paper, [7] derived an observational scaling relation between the large frequency separation and the mean density of several $\delta$ Scuti stars in eclipsing binary systems, in agreement with previous theoretical predictions [8]. Such large separations have also been used to create echelle diagrams $[9,10]$. However, no firm identification has been obtained for individual modes.

As such, it is necessary to develop ways to observationally constrain mode identification, in addition to searching for patterns in the observed frequency spectra. In slowly rotating stars, amplitude ratios and phases dif-

\footnotetext{
^e-mail: daniel.reese@obspm.fr
}

ferences based on multi-colour photometry, as well as line profile variations observed via spectroscopy, have proven to be popular methods for constraining mode identification $[11,12]$. Various efforts have been made to extend these methods to rapid rotators, but it is only recently that all of the necessary ingredients are starting to come together. These include:

1. rapidly rotating models in which the energy conservation equation is satisfied. Indeed, this is important for carrying out self-consistent non-adiabatic pulsation calculations as will be described in the following point. Currently the only code able to produce such models is the ESTER ${ }^{1}$ project $^{2}$ [13-15].

2. non-adiabatic pulsation calculations which fully take into account the effects of rapid rotation. Indeed, these can be used to predict which modes are excited by the $\kappa$-mechanism, the predominant mode-excitation mechanism in rapid rotators. Furthermore, only such calculations provide realistic pulsation-induced surface effective temperature variations, a key ingredient in photometric and spectroscopic mode signatures $[11,16]$. Up until recently, the only non-adiabatic code which fully takes into account the effects of rotation was that of [17]. However, this code was generally applied with a limited number of spherical harmonics to models

\footnotetext{
1 "Evolution STEllaire en Rotation"

${ }^{2}$ We note that the Self-Consistent Field method only solves the energy conservation equation after the averaging the model over level surfaces.
} 
based on a Chandrasekhar expansion, or even spherical models. This has, however, changed with the advent of the non-adiabatic version of the TOP code $[18,19]$, currently the only code which is set up to calculate pulsation modes in ESTER models.

3. detailed calculations of photometric mode visibilities and spectroscopic line profile variations (LPVs). Recently, [20] carried out detailed mode visibility calculations in rapidly rotating SCF models [21]. However these calculations, now need to be extended to the non-adiabatic case as described above. Probably the most realistic LPV calculations in rapidly rotating stars are those of [22], but were nonetheless carried out using the traditional approximation. Other calculations [23] fully include the effects of rotation on pulsations but use a simplified modelling of spectroscopic effects.

In the following, we describe the latest non-adiabatic pulsation calculations in ESTER models using the TOP code (Section 2), and some of the results for modeexcitation, mode visibilities, and line profile variations (Section 3).

\section{Theory}

\subsection{ESTER models}

The results presented in the following sections are based on $9 \mathrm{M}_{\odot}$ ESTER models with rotation rates ranging from 0.0 to $0.8 \Omega_{\mathrm{K}}$, where $\Omega_{\mathrm{K}}$ is the Keplerian break-up rotation rate. These models have a metallicity of $Z=0.025$ and use OPAL opacities. They harbour $\beta$ Cep type pulsations, in the $\mathrm{p}$ and g-mode range, which are excited by the iron opacity bump at $\log (T)=5.3$.

Models from the ESTER project fully take into account the effects of rotation. These include: centrifugal deformation, gravity darkening, and baroclinic effects which result from the mismatch between isobars and isotherms (given that the temperature profile is determined via the energy conservation equation). These effects lead to meridional circulation as well as differential rotation which depends both on $r$ and $\theta$, as illustrated in Fig. 1. From a numerical point of view, the ESTER code uses a multi-domain spectral approach with Chebyshev polynomials in the radial direction, and spherical harmonics in the horizontal directions. The multi-domain approach is illustrated in Fig. 1.

\subsection{Non-adiabatic pulsations}

The set of non-adiabatic pulsation equations implemented in the TOP code are the perturbed continuity Equation, Euler's equation, Poisson's equation, the perturbed energy conservation equation, and the perturbed energy flux equa-

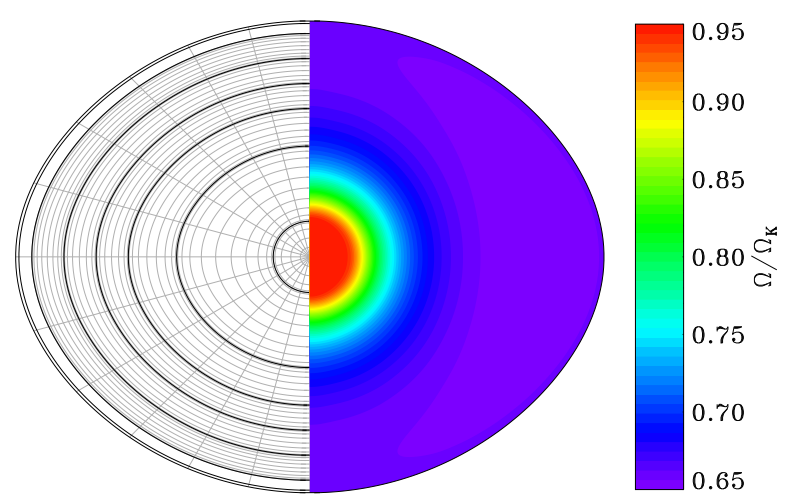

Figure 1: Multi-domain coordinate system and 2D rotation profile in an ESTER model.

tion:

$$
\begin{aligned}
0= & \frac{\delta \rho}{\rho_{0}}+\vec{\nabla} \cdot \vec{\xi} \\
0= & {[\omega+m \Omega]^{2} \vec{\xi}-2 i \vec{\Omega} \times[\omega+m \Omega] \vec{\xi} } \\
& -\vec{\Omega} \times(\vec{\Omega} \times \vec{\xi})-\vec{\xi} \cdot \vec{\nabla}\left(\varpi \Omega^{2} \vec{e}_{\varpi}\right)-\frac{P_{0}}{\rho_{0}} \vec{\nabla}\left(\frac{\delta P}{P_{0}}\right) \\
& +\frac{\vec{\nabla} P_{0}}{\rho_{0}}\left(\frac{\delta \rho}{\rho_{0}}-\frac{\delta P}{P_{0}}\right)-\vec{\nabla} \Psi+\vec{\nabla}\left(\frac{\vec{\xi} \cdot \vec{\nabla} P_{0}}{\rho_{0}}\right) \\
& +\frac{\left(\vec{\xi} \cdot \vec{\nabla} P_{0}\right) \vec{\nabla} \rho_{0}-\left(\vec{\xi} \cdot \vec{\nabla} \rho_{0}\right) \vec{\nabla} P_{0}}{\rho_{0}^{2}} \\
0 & \Delta \Psi-4 \pi G\left(\rho_{0} \frac{\delta \rho}{\rho_{0}}-\vec{\xi} \cdot \vec{\nabla} \rho_{0}\right) \\
0= & -i[\omega+m \Omega] \rho_{0} T_{0} \delta S+\epsilon_{0} \rho_{0}\left(\frac{\delta \epsilon}{\epsilon_{0}}+\frac{\delta \rho}{\rho_{0}}\right) \\
& -\vec{\nabla} \cdot \delta \vec{F}+\vec{\xi} \cdot \vec{\nabla}\left(\vec{\nabla} \cdot \overrightarrow{F_{0}}\right)-\vec{\nabla} \cdot\left[(\vec{\xi} \cdot \vec{\nabla}) \vec{F}_{0}\right] \\
& \left.+\vec{\xi} \cdot \vec{\nabla}\left(\vec{\nabla} T_{0}\right)-\vec{\nabla}\left(\vec{\xi} \cdot \vec{\nabla} T_{0}\right)\right] \\
\delta \vec{F}^{\mathrm{R}}= & {\left[\left(1+\chi_{T}\right) \frac{\delta T}{T_{0}}+\chi_{\rho} \frac{\delta \rho}{\rho_{0}}\right] \vec{F}_{0}^{\mathrm{R}}-\chi_{0}\left[T_{0} \vec{\nabla}\left(\frac{\delta T}{T_{0}}\right)\right.}
\end{aligned}
$$

where $\rho$ is the density, $\vec{\xi}$ the Lagrangian displacement, $\omega$ the pulsation frequency, $m$ the azimuthal order, $\Omega$ the rotation profile (which depends on $r$ and $\theta$ ), $\vec{\Omega}$ the rotation vector (i.e. $\Omega \vec{e}_{z}$ ), $\varpi$ the distance from the rotation axis, $P$ the pressure, $\Psi$ the gravitational potential, $T$ the temperature, $S$ the entropy, $\epsilon$ the nuclear energy generation rate, $\vec{F}$ the energy flux, $\vec{F}^{\mathrm{R}}$ the radiative energy flux, $\chi$ the opacity, $\chi_{T}$ the logarithmic derivative of $\chi$ with respect to $T$, i.e. $\frac{\partial \chi}{\partial T}$, and $\chi_{\rho}$ the logarithmic derivative of $\chi$ with respect to $\rho$. Quantities with the subscript " 0 " correspond to background equilibrium quantities whereas those preceded by a $\delta$ are Lagrangian perturbations. We note that the last term in Euler's equation is specific to non-barotropic models. In addition to the above equations, perturbed equations of state and opacities must be included, as well as a variety of boundary conditions which ensure regularity of the solution in the centre, a non-divergent gravitational potential perturbation at infinity, and the appropriate behaviour of 
Table 1: Numerical cost for calculating non-adiabatic modes.

\begin{tabular}{ccccc}
\hline $\mathbf{N}_{\mathbf{r}}$ & $\mathbf{N}_{\mathbf{h}}$ & $\mathbf{R A M}(\mathbf{G b})$ & Time (min) & Num. proc. \\
\hline 400 & 10 & 3.5 & & \\
400 & 15 & 7.9 & & \\
400 & 20 & 13.4 & 5 & 4 \\
400 & 29 & 28.0 & 10 & 8 \\
400 & 40 & 52.7 & 22 & 8 \\
400 & 50 & 82.3 & 26 & 16 \\
\hline
\end{tabular}

the pressure and temperature perturbations at the surface. Overall, the system comprises 10 equations with 10 unknowns. The numerical cost in solving this system is quite high as shown in Table 1 which gives the computational times and RAM for various radial and horizontal resolutions for given numbers of processors.

Using a similar approach as in the adiabatic case, it is possible to derive an integral expression for the frequency (but which will not benefit from a variational principle), as well as for the damping rate. The latter is known as the work integral and is used to increase the accuracy with which the damping rate is calculated. The relative difference between the numerical and integral values of the frequency is of the order of $10^{-4}$, whereas it is around $10^{-2}$ to $10^{-1}$ for the damping rate given its smaller value.

\section{Results}

\subsection{Frequencies and modes}

Figure 2 shows a typical frequency spectrum at $0.5 \Omega_{\mathrm{K}}$. The hollow diamonds correspond to modes which are excited via the $\kappa$-mechanism. For the same model, Fig. 3 shows the difference between adiabatic and non-adiabatic frequencies as a function of the damping rate. As expected, the largest frequency differences occur for the largest damping rates, i.e. where non-adiabatic effects are the strongest.

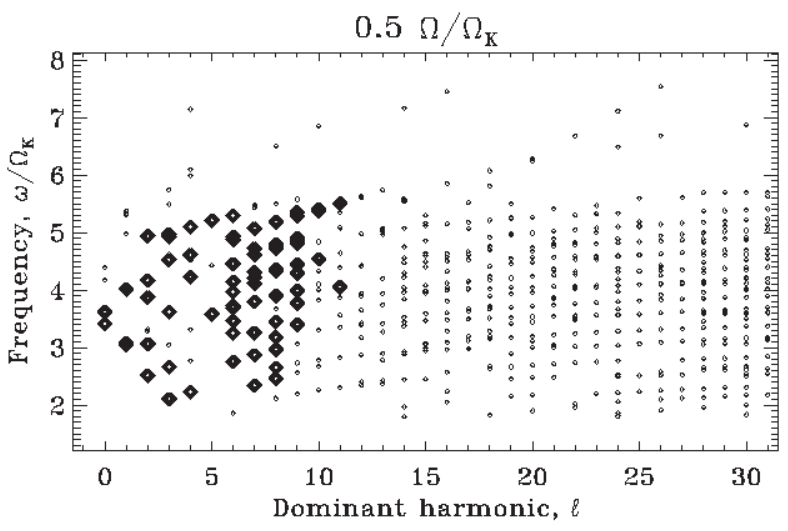

Figure 2: Frequency spectrum as a function of dominant spherical harmonic. The hollow diamonds correspond to unstable modes.

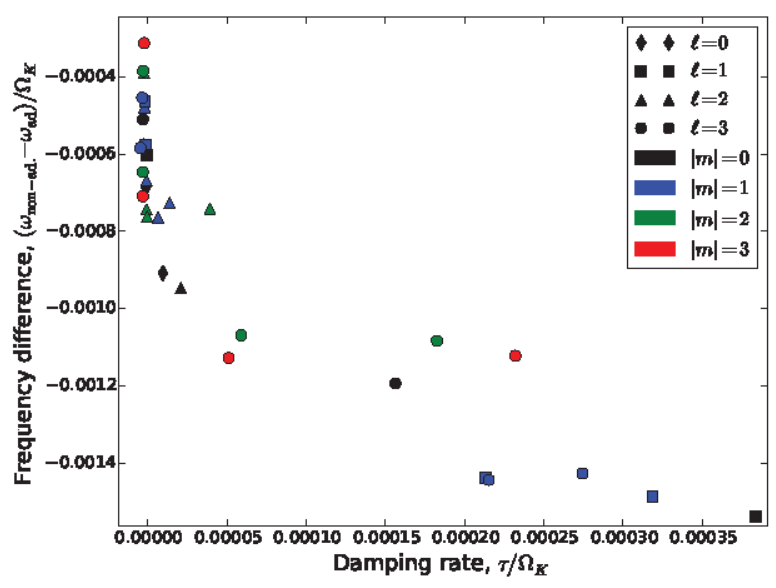

Figure 3: Difference between adiabatic and non-adiabatic frequencies as a function of the damping rate.

One can look more specifically at frequency multiplets. In Fig. 4, we show the evolution of various frequency multiplets as a function of the rotation rate. The thick lines correspond to excited modes. As can be seen, the prograde modes tend to remain excited longer, in agreement with the results in [24]. A look at the work integrals shows that modes are being stabilised by the very near surface layers, starting with the retrograde modes (see Fig. 2 of [25]).

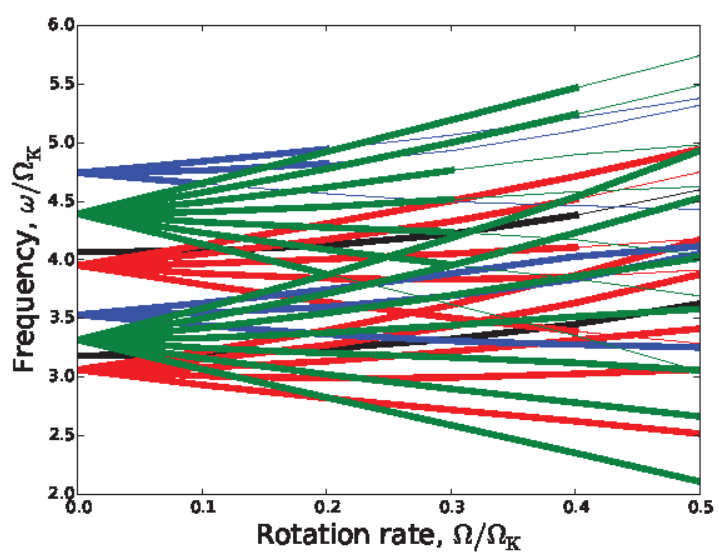

Figure 4: Frequencies multiplets as a function of the rotation rate. The thick lines corresponds to excited modes.

Different types of modes are excited. These include island modes and chaotic modes for the acoustic end of the spectrum [26], as well as mixed modes [27] and rosette modes [4], as illustrated in Fig. 5.

\subsection{Amplitude ratios}

Amplitude ratios were calculated for Strömgren and Johnson-Cousins photometric bands, and in the bolometric case using the formalism given in [20]. In order to calculate the intensity as a function of effective temperature, 

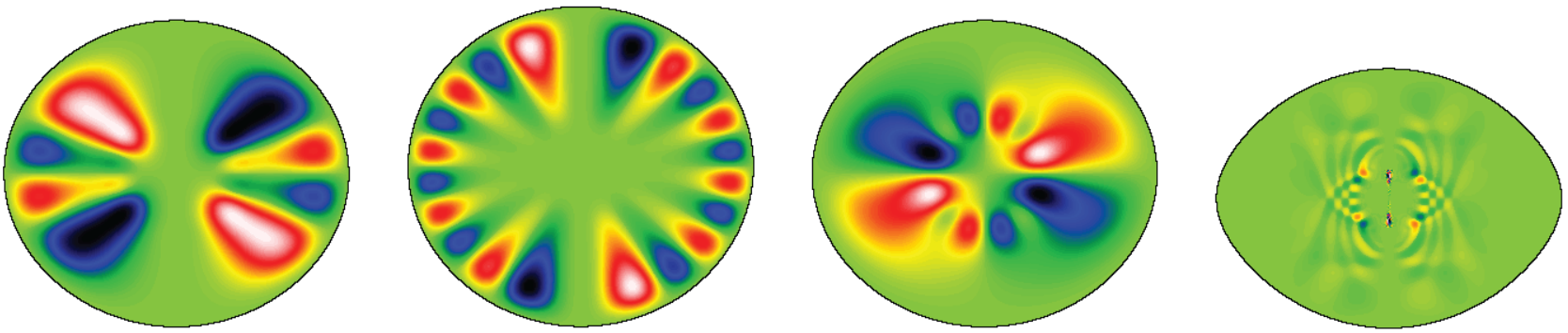

Figure 5: Different modes which are excited. These are, from left to right: an island mode, a whispering gallery mode, a mixed mode, and a rosette mode.

effective gravity, and orientation of the surface, we applied the following approximate formula:

$$
I\left(T_{\text {eff }}, g_{\text {eff }}, \mu\right)=I_{0}\left(T_{\text {eff }}\right) h\left(\mu, T_{\text {eff }}, g_{\text {eff }}\right)
$$

where $I_{0}\left(T_{\text {eff }}\right)$ was obtained from the integral of a blackbody spectrum weighted by the various photometric filter profiles, and $h\left(\mu, T_{\text {eff }}, g_{\text {eff }}\right)$ was obtained from [28], and $\mu=\cos \varphi$ where $\varphi$ is the angle between the line-of-sight and the normal to the surface. Figure 6 shows the resultant function obtained for the Strömgren u filter for a star at $0.8 \Omega_{\mathrm{K}}$. The decrease as a function of colatitude corresponds to gravity darkening, whereas the increase as a function of $\mu$ values is caused by limb darkening.

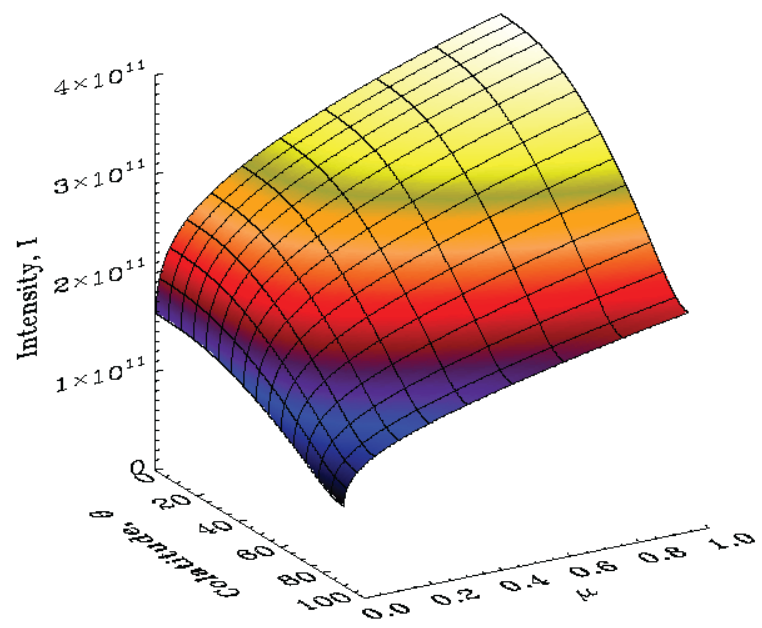

Figure 6: Intensity in the Strömgren photometric band as a function of the colatitude and $\mu$ for a model rotating at $0.8 \Omega_{\mathrm{K}}$.

Figure 7 shows the surface Lagrangian displacement, $\xi_{r}$, and surface $\delta T_{\text {eff }} / T_{\text {eff }}$ profiles for a given pulsation mode calculated with and without the adiabatic approximation. In contrast to $\xi_{r}$, the $\delta T_{\text {eff }} / T_{\text {eff }}$ profiles show a nonnegligible imaginary part in the non-adiabatic case. This corresponds to a phase shift between the displacement and temperature variations, as expected in such a case. As also anticipated, the modulus of the effective temperature variations, $\left|\delta T_{\text {eff }}\right|$, is smaller in the non-adiabatic case. These illustrate the importance of taking non-adiabatic effects into account prior to calculate mode visibilities, amplitude ratios, and phase differences.
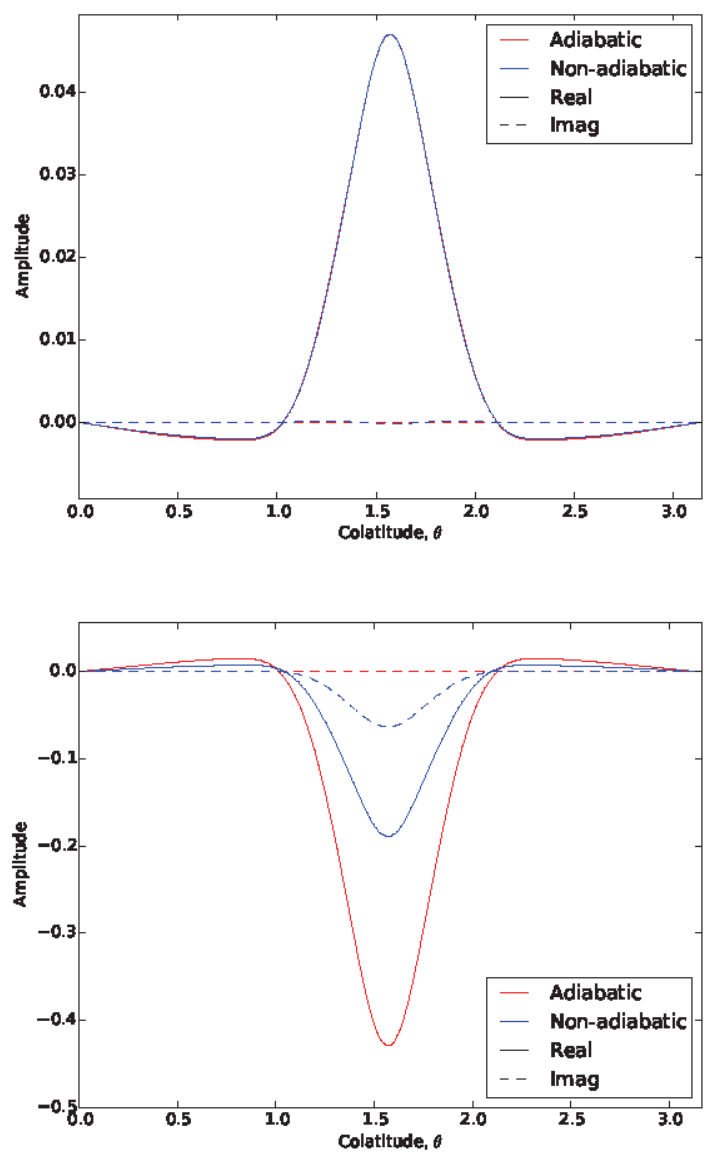

Figure 7: Surface vertical Lagrangian displacement (top panel) and surface $\delta T_{\text {eff }} / T_{\text {eff }}$ profiles (lower panel) for a given mode calculated with and without the adiabatic approximation.

Figure 8 shows a set of amplitude ratios for a given multiplet for a model with an inclination of $i=30^{\circ}$, and for a given mode at 4 different inclinations. In full agreement with previous results $[29,30]$, amplitude ratios become dependant both on the azimuthal order, $m$, and on the inclination as rotation is introduced. This, of course, complicates mode identification using amplitude ratios al- 
though we do note that [31] proposed an alternate mode identification strategy in such stars.
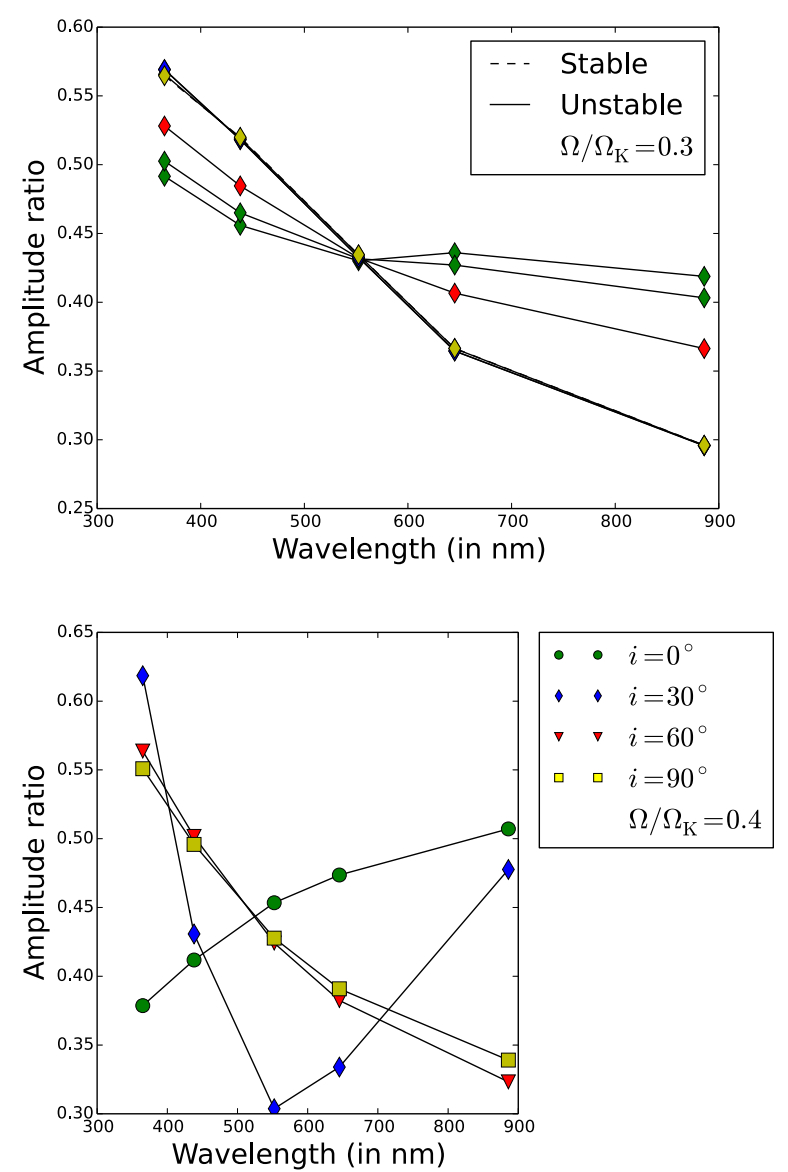

Figure 8: Amplitude ratios for an $\ell=3$ multiplet at an inclination of $i=30^{\circ}$ (upper panel) and for an $(\ell, m)=(2,0)$ mode at 4 different inclinations (lower panel).

\subsection{Line profile variations (LPVs)}

Another way of constraining mode identification is through LPVs. In the present case, we reuse some of the formulas from [20] with an additional Doppler term from both the stellar rotation and the oscillatory movements. The ray profile is modelled in crude way using a blackbody spectrum (thereby accounting for gravity darkening) as a background and a Gaussian profile as an intrinsic absorption ray. Furthermore, the contributions of $\delta T_{\text {eff }}$ and $\delta g_{\text {eff }}$ are neglected as are any dependence of the absorption ray on $T_{\text {eff }}$ and $g_{\text {eff }}$. A rudimentary description of limb-darkening is also included.

Figures 9 and 10 show two examples of LPVs calculated in a model rotating at $0.5 \Omega_{\mathrm{K}}$. These plots also contain the first and second moments, the amplitudes and phases of the first three harmonics of the variations for a particular Doppler position, and a meridional cross-section of the modes. A note worthy feature is that most of the variations take place in the wings of the absorption ray, which is different than what takes place in the non-rotating case. This feature is fairly frequent at rapid rotations and has been suggested as a possible explanation for macroturbulence in massive stars (Aerts, private communication).

\section{Conclusions}

The ability to carry out non-adiabatic pulsation calculations in rapidly rotating stars is an important step forward in being able to understand and interpret seismic data for such stars. Indeed, it is now possible to predict which modes are excited, and it will lead to more realistic predictions for amplitude ratios, phase differences, and line profile variations. The next steps forward include: gaining a better understanding of how rotation interacts with non-adiabatic effects, understanding what causes the differences between prograde and retrograde modes, including a realistic atmosphere in the stellar model when calculating amplitude ratios and line profile variations, and identifying modes in observed spectra using multi-colour photometry and spectroscopy.

\section{Acknowledgements}

DRR acknowledges financial support from the "Programme National de Physique Stellaire" (PNPS) of CNRS/INSU, France.

\section{References}

[1] M.J. Goupil, M.A. Dupret, R. Samadi, T. Boehm, E. Alecian, J.C. Suarez, Y. Lebreton, C. Catala, JA\&A 26, 249 (2005)

[2] T.R. Bedding, S.J. Murphy, I.L. Colman, D.W. Kurtz, Échelle diagrams and period spacings of $g$ modes in $\gamma$ Doradus stars from four years of Kepler observations, in European Physical Journal Web of Conferences (2015), Vol. 101 of European Physical Journal Web of Conferences, p. 01005

[3] T. Van Reeth, A. Tkachenko, C. Aerts, A\&A 593, A120 (2016)

[4] J. Ballot, F. Lignières, V. Prat, D.R. Reese, M. Rieutord, 2D Computations of g-modes in Fast Rotating Stars, in Progress in Solar/Stellar Physics with Helio- and Asteroseismology, edited by H. Shibahashi, M. Takata, A.E. Lynas-Gray (2012), Vol. 462 of Astronomical Society of the Pacific Conference Series, p. 389

[5] M.P. Bouabid, M.A. Dupret, S. Salmon, J. Montalbán, A. Miglio, A. Noels, MNRAS 429, 2500 (2013)

[6] R.M. Ouazzani, S.J.A.J. Salmon, V.L. Antoci, T.R. Bedding, S.J. Murphy, I.W. Roxburgh, MNRAS (2016), in press, astro-ph.SR:1610.06184

[7] A. García Hernández, S. Martín-Ruiz, M.J.P.F.G. Monteiro, J.C. Suárez, D.R. Reese, J. PascualGranado, R. Garrido, 811, L29 (2015)

[8] D. Reese, F. Lignières, M. Rieutord, A\&A 481, 449 (2008) 


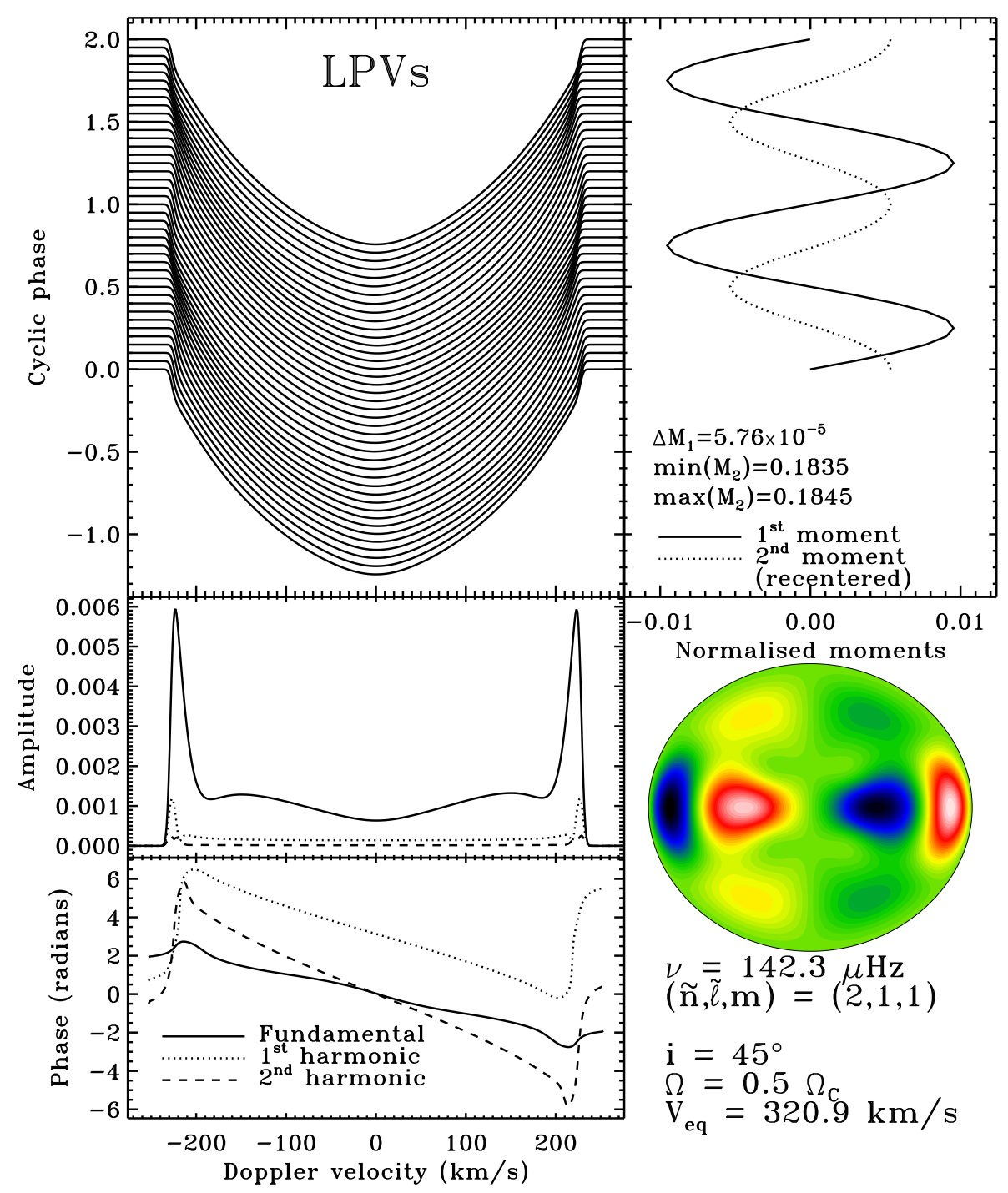

Figure 9: Line profile variations for an $(n, \ell, m)=(1,3,1)$ mode (top left panel), first and second moments (top right panel), amplitudes and phases of the first three harmonics of the variations across the line profile (lower left panels), and meridional cross-section of the mode (lower right panel).

[9] A. García Hernández, A. Moya, E. Michel, J.C. Suárez, E. Poretti, S. Martín-Ruíz, P.J. Amado, R. Garrido, E. Rodríguez, M. Rainer et al., A\&A 559, A63 (2013)

[10] M. Paparó, J.M. Benkő, M. Hareter, J.A. Guzik, ApJS 224, 41 (2016)

[11] M.A. Dupret, J. De Ridder, P. De Cat, C. Aerts, R. Scuflaire, A. Noels, A. Thoul, A\&A 398, 677 (2003)

[12] W. Zima, D. Wright, J. Bentley, P.L. Cottrell, U. Heiter, P. Mathias, E. Poretti, H. Lehmann, T.J. Montemayor, M. Breger, A\&A 455, 235 (2006)

[13] M. Rieutord, F. Espinosa Lara, Ab Initio Modelling of Steady Rotating Stars, in Lecture Notes in Physics, Berlin Springer Verlag, edited by M. Goupil, K. Belkacem, C. Neiner, F. Lignières, J.J. Green (2013), Vol. 865 of Lecture Notes in Physics, Berlin
Springer Verlag, p. 49

[14] F. Espinosa Lara, M. Rieutord, A\&A 552, A35 (2013)

[15] M. Rieutord, F. Espinosa Lara, B. Putigny, Journal of Computational Physics 318, 277 (2016)

[16] M. Dupret, J. De Ridder, C. Neuforge, C. Aerts, R. Scuflaire, A\&A 385, 563 (2002)

[17] U. Lee, I. Baraffe, A\&A 301, 419 (1995)

[18] D. Reese, F. Lignières, M. Rieutord, A\&A 455, 621 (2006)

[19] D.R. Reese, K.B. MacGregor, S. Jackson, A. Skumanich, T.S. Metcalfe, A\&A 506, 189 (2009)

[20] D.R. Reese, V. Prat, C. Barban, C. van 't VeerMenneret, K.B. MacGregor, A\&A 550, A77 (2013)

[21] K.B. MacGregor, S. Jackson, A. Skumanich, T.S. Metcalfe, ApJ 663, 560 (2007)

[22] R.H.D. Townsend, MNRAS 284, 839 (1997) 


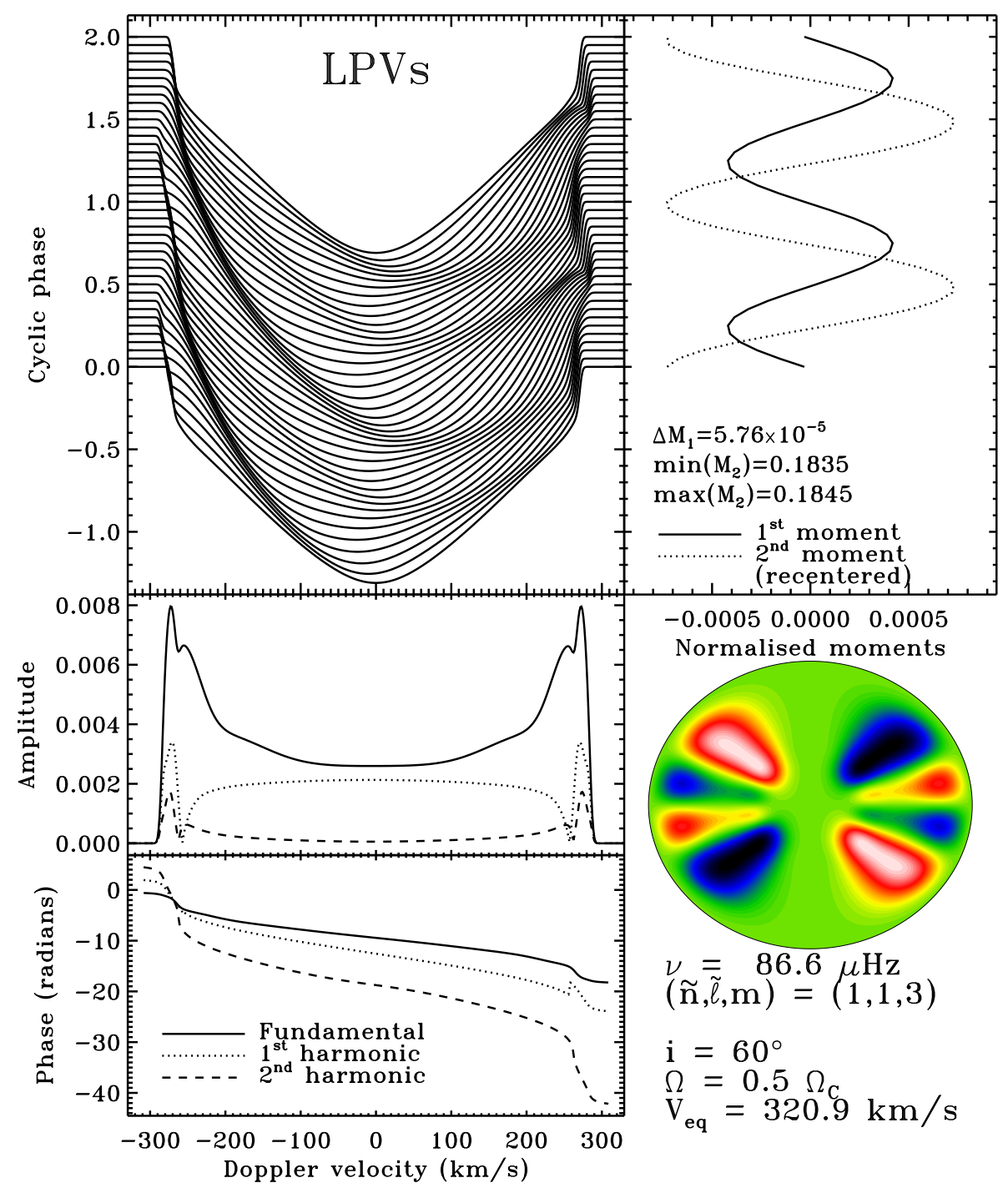

Figure 10: Same as Fig. 9 but for the $(n, \ell, m)=(0,6,3)$ mode.

[23] M.J. Clement, Pulsation in Rapidly Rotating Stars, in IAU Symp. 162: Pulsation; Rotation; and Mass Loss in Early-Type Stars, edited by L.A. Balona, H.F. Henrichs, J.M. Le Contel (1994), p. 117

[24] U. Lee, Pulsational Stability of Rotating Stars, in American Institute of Physics Conference Series, edited by V. Mioc, C. Dumitrche, N.A. Popescu (2008), Vol. 1043 of American Institute of Physics Conference Series, pp. 344-350

[25] D.R. Reese, Internal rapid rotation and its implications for stellar structure and pulsations, in European Physical Journal Web of Conferences (2015), Vol. 101 of European Physical Journal Web of Conferences, p. 05007

[26] F. Lignières, B. Georgeot, A\&A 500, 1173 (2009)
[27] R.M. Ouazzani, I.W. Roxburgh, M.A. Dupret, A\&A 579, A116 (2015)

[28] A. Claret, A\&A 363, 1081 (2000)

[29] J. Daszyńska-Daszkiewicz, W.A. Dziembowski, A.A. Pamyatnykh, M.J. Goupil, A\&A 392, 151 (2002)

[30] R.H.D. Townsend, MNRAS 343, 125 (2003)

[31] D.R. Reese, F. Lignières, J. Ballot, V. Prat, C. Barban, C. van't Veer-Menneret, K.B. MacGregor, Mode Visibilities and Frequency Patterns in Rapidly Rotating Stars, in Progress in Physics of the Sun and Stars: A New Era in Helio- and Asteroseismology, edited by H. Shibahashi, A.E. Lynas-Gray (2013), Vol. 479 of Astronomical Society of the Pacific Conference Series, p. 545 